\section{POS1096 THE EFFECT OF ONE DRY NEEDLING SESSION ON PAIN AND CENTRAL PAIN PROCESSING IN PATIENTS WITH KNEE OSTEOARTHRITIS: A RANDOMIZED CONTROLLED TRIAL}

S. Vervullens ${ }^{1}$, L. Meert ${ }^{1}$, I. Baert ${ }^{1}$, N. Delrue ${ }^{2}$, K. Heusdens ${ }^{3}$, A. Hallemans ${ }^{1}$, T. Van Criekinge ${ }^{1,4}$, R. Smeets ${ }^{5,6}, K$. De Meulemeester on behalf of MOVANT (MOvement ANTwerp) of the University of Antwerp. ${ }^{1}$ Antwerp University, Rehabilitation Sciences and Physiotherapy, Antwerp, Belgium; ${ }^{2}$ Dry Needling Ghent, Rehabilitation Sciences, Ghent, Belgium; ${ }^{3}$ University Hospital of Antwerp, Orthopedics, Antwerp, Belgium; ${ }^{4}$ KULeuven, Rehabilitation Sciences, Bruges, Belgium; ${ }^{5}$ Maastricht University, Rehabilitation Medicine, Maastricht, Netherlands; ${ }^{6}$ University Hospital of Maastricht, Rehabilitation Medicine, Maastricht, Netherlands; ${ }^{7}$ Ghent University, Rehabilitation Sciences, Ghent, Belgium

Background: Osteoarthritis $(\mathrm{OA})$ is the leading cause of chronic disability in the elderly ${ }^{1,2}$. Abnormal central pain processing (CPP) is present in around $30 \%$ of the knee OA patients ${ }^{3}$ and can be partly induced by peripheral nociception through long term potentiation ${ }^{4}$. An attempt to resolve abnormal CPP can be to eliminate this nociception ${ }^{5}$. Myofascial trigger points (MTrPs) are often present in knee $\mathrm{OA}^{6}$, can lead to nociception ${ }^{7}$ and therefore abnormal CPP if prolonged present ${ }^{8}$. These are usually defined as hypersensitive tender spots within taut bands of a muscle ${ }^{9}$. Additionally, both MTrPs and knee OA can induce disturbed motor control, increased co-antagonist activation and modified gait pattern ${ }^{10,11}$. Dry needling (DN) is often used to deactivate the MTrP and thus resolve the source of nociception, which normally results in reducing pain and restoring muscle dysfunction ${ }^{12}$. However, studies about the effect of DN on CPP and other outcomes than pain are very limiting. Therefore, more high-quality studies concerning DN and its effects on CPP, muscle features and gait are needed ${ }^{13,14}$.

Objectives: The aim of this randomized controlled trial is to assess the effect of one DN session compared to one sham needling (SN) session on pain (processing), muscle activity and gait in patients with knee $O A$.

Methods: 61 patients participated of which 31 were allocated to the DN and 30 to the SN group. Each patient underwent one treatment session. Primary outcomes were pain intensity, measured with questionnaires; and CPP, measured with quantitative sensory testing. Secondary outcomes included muscle co-activation, measured with electromyography; and spatiotemporal parameters, measured with gait analysis. Patients were assessed at baseline, 15 minutes (post 1) and 3 days after intervention (post2- only for the outcome pain). Linear mixed models was used to identify the possible differences over time between the two therapy modalities.

Results: The following significant within group differences were observed: decreased pain, stride- and step time and increased widespread pain pressure threshold and step length. A significant between group difference of the conditioned pain modulation score was found, whereas the $\mathrm{SN}$ group showed a decrease in difference between the pain pressure threshold scores (with and without conditioning stimulus) and the DN group remained stable. No other significant between or within group differences were detected. However, if we compared both interventions, the change over time for the visual analogue scale (VAS) behaved different in the DN ( $p<0.05$, post 2 - baseline) and $S N$ group $(p<0.05$, post 1 - baseline and post 2 - post 1$)$.

Conclusion: One DN session has no larger effect on all outcome measurements compared to SN. Both therapies seem to be useful to improve pain and

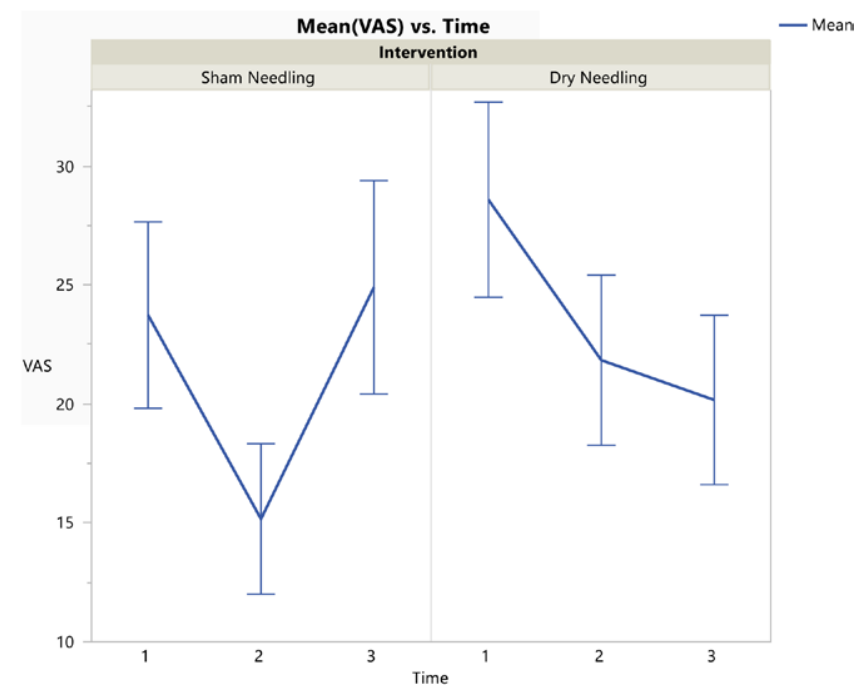

Figure 1. Mean and standard error of VAS scores over time in the DN and SN group widespread pain pressure threshold in short term, however the improvement of pain differs in the groups. Although improvements in some spatiotemporal parameters were observed, it is uncertain they are of clinical relevance or related to treatment. More research is necessary to reveal the ideal number of sessions of DN to improve outcomes and to reveal the effect of DN compared to no treatment, as SN could have hide the real treatment effect.

REFERENCES:

[1] Reginster, J. Y. Rheumatol. Oxf. 41 Supp 1, 3-6 (2002)

[2] Lespasio, M. J. et al. Perm J 21, 16-183 (2017)

[3] Lluch, E. et al. Eur J Pain 18, 1367-75 (2014)

[4] Mease, P. J. et al. J. Rheumatol. 38, 1546-1551 (2011)

[5] Nijs, J. et al. Expert Opin Pharmacother 15, 1671-83 (2014)

[6] Bajaj, P. et al. J. Musculoskelet. Pain 9, 17-33 (2001)

[7] Jafri, M. S. Int. Sch. Res. Not. 2014, (2014)

[8] Cagnie, B. et al. Curr Pain Headache Rep 17, 348 (2013)

[9] Borg-Stein, J. et al. Arch. Phys. Med. Rehabil. 83, S40-S47 (2002)

[10] Childs, J. D. et al. Clin. Biomech. 19, 44-9 (2004)

[11] Ibarra, J. M. et al. J Pain 12, 1282-8 (2011)

[12] Rahou-El-Bachiri, Y. et al. J. Clin. Med. 9, (2020)

[13] Stieven, F. F. et al. J. Manipulative Physiol. Ther. (2021)

[14] Dor, A. et al. J Bodyw Mov Ther 21, 642-647 (2017)

Acknowledgements: I like to thank and acknowledge the contribution of the other executive researchers (Lise Brosens, Ayoub El Bouchaoni, Ben Ceusters, Sven Huybrechts and Mathias Van Loon), the participated dry needling therapists, Dry Needling Ghent, Trigger and the University Hospital of Antwerp.

Disclosure of Interests: None declared.

DOI: 10.1136/annrheumdis-2021-eular.2108

\begin{tabular}{|l|l}
\hline POS1097 & TREATMENT KNOWLEDGE AND PREFERENCES \\
& FOR BLACK PEOPLE WITH HIP AND KNEE \\
& OSTEOARTHRITIS: A SYSTEMATIC LITERATURE \\
& REVIEW AND META-ANALYSIS
\end{tabular}

C. Brantner ${ }^{1}$, D. Pearce-Fisher ${ }^{1}$, C. Moezinia ${ }^{1}$, H. Tornberg ${ }^{1}$, J. Fitzgerald ${ }^{2,3}$, M. Parks ${ }^{4,5}$, P. Sculco ${ }^{4,5}$, C. Kahlenberg ${ }^{4}$, C. Mensah ${ }^{1}$, A. Premkuar ${ }^{4}$, N. Williams ${ }^{6}$, M. Demetres ${ }^{7}$, S. Goodman ${ }^{8,9} .{ }^{1}$ Hospital for Special Surgery, Medicine, New York, United States of America; ${ }^{2}$ Ronald Reagan UCLA Medical Center, Medicine, Los Angeles, United States of America; ${ }^{3}$ UCLA Santa Monica Medical Center, Medicine, Santa Monica, United States of America; ${ }^{4}$ Hospital for Special Surgery, Orthopedic Surgery, New York, United States of America; ${ }^{5}$ Weill Cornell Medicine, Orthopedic Surgery, New York, United States of America; ${ }^{6}$ Weill Cornell Medicine, Population Health Sciences, New York, United States of America; ${ }^{7}$ Weill Cornell Medicine, Information Technologies and Services - Library, New York, United States of America; ${ }^{8} \mathrm{Hospital}$ for Special Surgery, Rheumatology, New York, United States of America; ${ }^{9}$ Weill Cornell Medicine, Rheumatology, New York, United States of America

Background: Black people are less likely to undergo total joint arthroplasties despite reporting more severe symptoms. (1) While racial disparities exist in treatment utilization for osteoarthritis, comprehensive studies of the treatment preferences of Black people have not been conducted.

Objectives: The purpose of this manuscript is to systematically review the liter ature and identify Black osteoarthritis patients' treatment preferences to understand how they may contribute to racial differences in the utilization of different treatment options.

Methods: Searches ran on April 8, 2019 and April 7, 2020 in the following databases: Ovid MEDLINE (ALL - 1946 to Present); Ovid EMBASE (1974 to present); and The Cochrane Library (Wiley). Using the Patient/Population-Intervention-Comparison/Comparator-Outcome (PICO) format, our population of interest was Black people with hip and/or knee osteoarthritis, our intervention was preferences and opinions about treatment options for osteoarthritis, our comparator was white people with hip and/or knee osteoarthritis, and our outcome was preferences of osteoarthritis therapies. The protocol was registered under the PROSPERO international register, and the Preferred Reporting Items of Systematic reviews and Meta-Analyses (PRISMA) guidelines were followed. Results: Searches across the chosen databases retrieved 10,894 studies after de-duplication, 182 full text, and 31 selected for inclusion in this review. Black people were less likely to use NSAIDs or narcotic analgesics compared to white people. (Figure 1) Black people were more likely than white people to use spirituality and prayer, as well as topical treatments. Utilization of meditation, supplement/vitamin use, and hot/cold treatments was not significantly different between groups. Black people were less willing than white people to consider or undergo joint replacements, even if the procedure was needed and recommended by a physician.

Conclusion: Racial differences persist in OA care across the spectrum of options. Future interventions should focus on providing accessible information surrounding treatment options and targeting perceptions of the importance of joint health. 
REFERENCES:

[1] Suarez-Almazor ME, Souchek J, Kelly PA, et al. Ethnic Variation in Knee Replacement: Patient Preferences or Uninformed Disparity? Arch Intern Med. 2005;165(10):1117-1124. doi:10.1001/archinte.165.10.1117

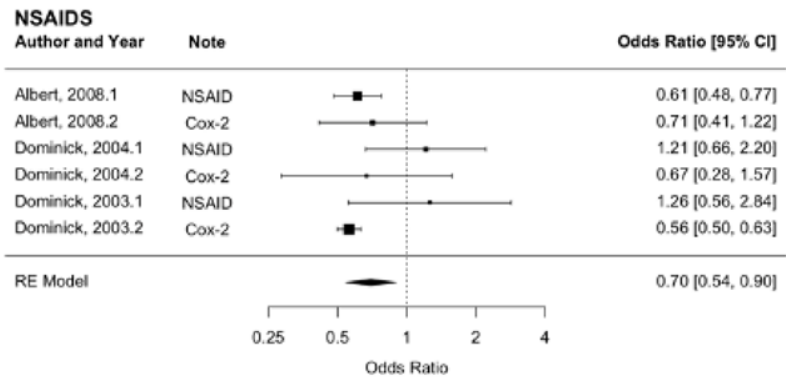

\section{Narcotic Analgesics}

Author and Year

Odds Ratio $[95 \% \mathrm{Cl}]$

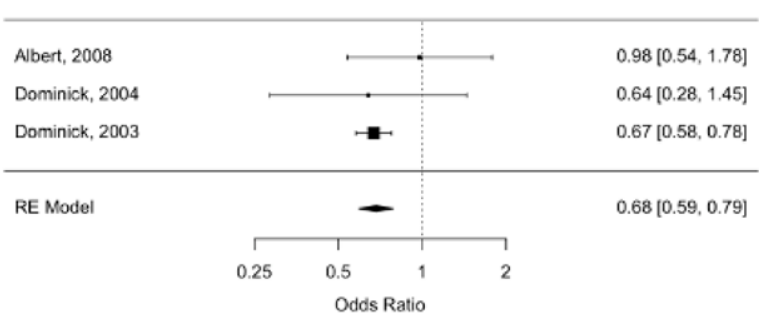

$\mathrm{RE}=$ Random Effects; NSAIDs $=$ Non-steroidal anti-inflammatory drugs COX-2 = Cyclo-oxygenase 2 inhibitors; $\mathrm{Cl}=$ Confidence Interval

Figure 1. Meta-analysis describing the odds ratios of Black people using NSAIDs and Narcotic Analgesics compared to white people

Disclosure of Interests: Collin Brantner: None declared, Diyu Pearce-Fisher: None declared, Carine Moezinia: None declared, Haley Tornberg: None declared, John FitzGerald: None declared, Michael Parks Consultant of: Zimmer Biomet, Peter Sculco Consultant of: EOS Imaging, Intellijoint Surgical, DePuy Synthes, Lima Corporate, Cynthia Kahlenberg: None declared, Curtis Mensah: None declared, Ajay Premkuar: None declared, Nicholas Williams: None declared, Michelle Demetres: None declared, Susan Goodman Consultant of: UCB, Grant/ research support from: Novartis, Horizon Therapeutics.

DOI: 10.1136/annrheumdis-2021-eular.3136

\section{POS1098 PREVALENT COMORBIDITIES ASSOCIATED WITH CLINICALLY DIAGNOSED OSTEOARTHRITIS: A CASE- CONTROL ANALYSIS INCLUDING 1,936,792 PEOPLE}

$\underline{\text { A. Vivekanantham }}^{1,2}$, D. Prieto-Alhambra ${ }^{1,3}$, D. E. Robinson ${ }^{1} .{ }^{1}$ University of Oxford, Centre for Statistics in Medicine, Nuffield Department of Orthopaedics, Rheumatology, and Musculoskeletal Sciences, Oxford, United Kingdom; ${ }^{2}$ Nuffield Orthopaedic Centre, Rheumatology, Oxford, United Kingdom; ${ }^{3}$ Erasmus University Medical Center, Health Data Sciences, Rotterdam, Netherlands

Background: Osteoarthritis $(\mathrm{OA})$ is the most common form of arthritis and a major cause of pain and disability worldwide. Individuals with OA have increased cardiovascular morbidity and mortality, and other comorbidities are also more common amongst them than in the general population. In this study, we examined the prevalence and timing of the diagnosis of co-morbidities prior to the clinical diagnosis of OA. Objectives: To determine the odds of comorbidities in newly diagnosed OA cases versus matched controls in the up to 10 years prior to diagnosis.

Methods: Case-control study of people registered in the Information System for Research in Primary Care (SIDIAP). SIDIAP includes primary care records covering over $80 \%$ of the population of Catalonia, Spain.

Participants with an incident diagnosis of OA, based on ICD-10-CM disease codes, were matched to up to 4 controls by age (within 2 years), gender and primary care practice. The first diagnosis date of OA used the index date, with matched controls using the same index date, to retrospectively review for comorbidities. Patients were required to have at least 3 years continuous registration prior to the index date. A total of 57 comorbidities were considered, based on prior knowledge and clinical consensus.

Descriptive statistics were used to obtain the demographic information about cases and controls. Counts of any comorbidity were calculated, and univariable and multivariable logistic regression were used to calculate odds ratios (ORs) and $95 \%$ confidence intervals (Cls) of individual comorbidities adjusted for age, gender and socio-economic status.

Results: In total, there were 455,494 OA cases and 1,481,298 matched controls. The cases and controls were similar with regards to age, gender and deprivation level. The incident diagnosis of any comorbidity at any time prior to index date was $77 \%(n=350,913)$ in cases versus $61 \%(n=909,497)$ in controls.

The results from the multivariable analysis [Table 1] showed that OA patients had higher prevalence of many comorbidities up to 1 year prior to their diagnosis of OA compared to up to 10 years prior to diagnosis. They were most likely to be diagnosed with another musculoskeletal condition followed by neuro-psychiatric and metabolic and cardiovascular conditions. Conversely, patients with OA were less likely to be diagnosed with cancer up to 10 years prior to index date than matched controls. Conclusion: Patients with $\mathrm{OA}$ have multiple chronic conditions. Our results found that the diagnosis of these other co-morbidities (particularly musculoskeletal and neuro-psychiatric conditions) were more likely to occur in the 1-year prior to their diagnosis of OA, compared to in the 10-year prior, except for lymphoma and solid malignancy. These results help us to further understand the relationship and timing of the development of multiple co-morbidities in patients with OA

Table 1. Multivariable analysis at $\mathbf{1}$ year and $\mathbf{1 0}$ year prior to index date

\begin{tabular}{lcc} 
Co-morbidity & $\begin{array}{c}\text { Multivariable analysis } \\
1 \text {-year prior } \\
(\mathrm{OR}, 95 \% \mathrm{Cl})\end{array}$ & $\begin{array}{c}\text { Multivariable analysis } \\
10 \text {-year prior }(\mathrm{OR}, \\
95 \% \mathrm{Cl})\end{array}$ \\
\hline $\begin{array}{l}\text { Musculoskeletal conditions } \\
\text { Ankylosing Spondylosis }\end{array}$ & $3.12(2.41,4.05)$ & $1.27(1.12,1.43)$ \\
Fibromyalgia & $4.24(3.96,4.54)$ & $1.85(1.80,1.91)$ \\
Rheumatoid Arthritis & $2.67(2.40,2.97)$ & $1.27(1.20,1.34)$ \\
Back/ neck pain & $2.41(2.38,2.45)$ & $1.76(1.75,1.78)$ \\
Neuro-psychiatric & & \\
Anxiety & $1.69(1.65,1.73)$ & $1.23(1.22,1.24)$ \\
Depression & $1.87(1.81,1.93)$ & $1.25(1.24,1.27)$ \\
Irritable Bowel Syndrome & $1.90(1.71,2.11)$ & $1.28(1.22,1.34)$ \\
Migraine & $1.81(1.69,1.94)$ & $1.23(1.20,1.27)$ \\
Cancer & & \\
Leukaemia & $1.07(0.88,1.30)$ & $0.91(0.82,1.00)$ \\
Lymphoma & $0.84(0.68,1.03)$ & $0.90(0.82,0.99)$ \\
Solid malignancy & $0.95(0.91,0.99)$ & $0.95(0.93,0.97)$ \\
Other medical conditions & & \\
Stroke & $1.15(1.10,1.20)$ & $0.95(0.93,0.97)$ \\
Hypertension & $1.70(1.68,1.74)$ & $1.26(1.25,1.28)$ \\
Diabetes & $1.34(1.30,1.38)$ & $1.07(1.06,1.09)$ \\
Obesity & $1.96(1.92,2.01)$ & $1.60(1.58,1.62)$ \\
\hline
\end{tabular}

Disclosure of Interests: Arani Vivekanantham: None declared, Daniel Prieto-Alhambra: None declared, Danielle E Robinson Grant/research support from: Dr. Prieto-Alhambra reports grants and other from AMGEN, grants, non-financia support and other from UCB Biopharma, grants from Les Laboratoires Servier, outside the submitted work; and Janssen, on behalf of IMI-funded EHDEN and EMIF consortiums, and Synapse Management Partners have supported training programmes organised by DPA's department and open for external participants. DOI: 10.1136/annrheumdis-2021-eular.3202

\section{POS1099 EFFICACY OF INTRA-ARTICULAR CORTICOSTEROID INJECTIONS IN KNEE OSTEOARTHRITIS: A SYSTEMATIC REVIEW AND META-ANALYSIS OF RANDOMIZED CONTROLLED TRIALS}

A. Najm ${ }^{1}$, A. Alunno ${ }^{2}$, C. Weill ${ }^{3}$, J. Gwinnutt ${ }^{4}$, F. Berenbaum ${ }^{5} .{ }^{1}$ Institute of Infection, Immunity and Inflammation, College of Medical Veterinary and Life Sciences, University of Glasgow, Rheumatology, Glasgow, United Kingdom; ${ }^{2}$ University of Perugia, Rheumatology Unit, Department of Medicine, Perugia, Italy; ${ }^{3}$ Paris Descartes University, Bibliothèque interuniversitaire de Santé, Paris, France; ${ }^{4}$ Centre for Epidemiology Versus Arthritis, Centre for Musculoskeletal Research, School of Biological Sciences, Faculty of Biology, Medicine and Health, The University of Manchester, Division of Musculoskeletal and Dermatological Sciences, Manchester, United Kingdom; ${ }^{5}$ Sorbonne Université, INSERM CRSA Saint-Antoine, AP-HP, Saint Antoine Hospital, Paris, France. Rheumatology, Paris, France

Background: Knee osteoarthritis $(\mathrm{OA})$ is a frequent degenerative disease representing an important health and economic burden. Symptomatic medical treatments available include intra-articular (IA) injections of corticosteroids (GC) but 